\title{
LAS GRANDES LÍNEAS MAESTRAS DE LA HISTORIOGRAFÍA ARTÍSTICA CHILENA (1928-1965)
}

\author{
Albert Ferrer Orts \\ Universitat de València \\ Alberto Madrid Letelier \\ Universidad de Playa Ancha de Ciencias de la Educación
}

\begin{abstract}
Resumen: El presente estudio ofrece las líneas maestras de la historiografía del arte en Chile, las cuales se consolidaron definitivamente en las décadas centrales del siglo XX gracias a la formación académica y sensibilidad cultural de autores como Luis Álvarez Urquieta, Alfredo Benavides, Eugenio Pereira y Antonio Romera, además de Luis Cousiño, Alberto Mackenna, Tomás Lago y Pablo Vidor. Para ello, recogemos en este artículo los principales hitos bibliográficos de dichos teóricos. El trabajo viene también a complementar otras dos investigaciones sobre los orígenes de la historia del arte en Chile a través de sus precursores y del papel que desempeñaron sucesivamente en la Escuela de Artes y Oficios, la Academia de Pintura, la Escuela de Bellas Artes, la Sociedad Artística (después Unión Artística) y el Museo Nacional de Bellas Artes de Santiago entre los siglos XIX y XX.
\end{abstract}

Palabras clave: Historiografía del arte, Chile, Museo Nacional de Bellas Artes, pioneros.

The great master lines of Chilean artistic historiography (1928-1965)

Abstract: This study provides the outlines of the history of art in Chile, which was finally consolidated in the middle decades of the twentieth century through academic training and cultural sensitivity of its authors, such as Luis Álvarez Urquieta, Alfredo Benavides, Eugenio Pereira, Antonio Romera, plus Luis Cousino, Alberto Mackenna, Tomás Lago and Pablo Vidor. We take a journey through the main bibliographic milestone of these theorists. The work also complements two other investigations into the origins of art history in Chile through its precursors and the role played successively in the Escuela de Artes y Oficios, the Academia de Pintura, the Escuela de Bellas Artes, the Sociedad Artística (later Unión Artística) and the Museo Nacional de Bellas Artes in Santiago between the nineteenth and twentieth centuries.

Key words: Historiography of art, Chile, Museo Nacional de Bellas Artes, pioneers.

La preocupación por la historia del arte en Chile se manifiesta a través de diversas generaciones de estudiosos que, desde mediados del siglo XIX, fueron aportando informaciones y puntos de vista sobre cuestiones artísticas y estéticas relativas a su país. Su peso específico, sin embargo, estuvo supeditado a la historia en un escenario político y social en el que se hacía necesario dotar de significado identitario los primeros pasos de la nueva nación en el contexto internacional. Entre los primeros que de ello se ocuparon encontramos a historiadores y políticos como Diego Barros Arana, Miguel L. Amunátegui Aldunate o Benjamín Vicuña Mackenna, a los que siguieron artistas como José Miguel Blanco,

Data de recepció: 8 de juliol de 2016 / Data d'acceptació: 24 d'octubre de 2016. 
Pedro Lira y Vicente Grez y, más adelante, Richard Richon-Brunet, Luis Cousiño, Alberto Mackenna Subercaseaux y Luis Álvarez Urquieta.

Sin embargo, llegados a este punto cabe preguntarse: ¿cuándo nace la historiografía artística en Chile?, ¿quiénes fueron sus protagonistas?, y ¿qué grandes líneas maestras trazaron en adelante?

A decir verdad, el desarrollo de lo que podríamos denominar con propiedad historiografía del arte chileno se enmarca en las décadas centrales del siglo XX, entre finales de su primer tercio y las postrimerías del segundo. Dicho de otra forma, entre las obras de Luis Álvarez Urquieta y de Eugenio Pereira Salas se consolida en el país la bibliografía que sistemáticamente comienza a abordar la naturaleza de su peculiaridad artística en sus más diversas vertientes: pintura, escultura, arquitectura, orfebrería, urbanismo... en una época en la que todavía no existía la especialidad académica en formato teórico en sus universidades, más bien vinculada a las Bellas Artes e impartida regularmente por los propios artistas en su faceta docente.

Hasta ese momento, o período, no es que no se hubiera teorizado sobre el origen y las circunstancias del arte chileno, considerado como tal el producido desde la conquista española en adelante, toda vez que el arte precolombino y la artesanía pasaban progresivamente a formar parte de disciplinas hermanas como la arqueología y la antropología. Desde la primera generación de chilenos nacidos en período republicano, la preocupación por la historia, las costumbres y también el arte del nuevo país son una constante in crescendo, bien es cierto que cultivadas por el patriciado urbano mayormente, culto y viajado a la par que lógicamente impulsado por su espíritu nacionalista, que comprende grosso modo desde los trabajos del historiador Miguel L. Amunátegui hasta los del pintor y crítico Richard Richon-Brunet, es decir, el lapso de tiempo inmediatamente anterior al aquí tratado.

Objeto igualmente de esta aproximación lo serán también los esfuerzos llevados a cabo entre 1922 y 1930 por Luis Cousiño Talavera, Alberto Mackenna Subercaseaux, Tomás Lago Pinto y Pablo Vidor Doctor, a propósito de los inventarios que se realizan para el Museo Nacional de Bellas Artes (figura 1), los únicos publicados hasta el momento -junto al pequeño catálogo editado en 1977-, y la adquisición de la colección de réplicas escultóricas para el mismo, que si bien desbordan un tanto el marco cronológico propuesto no dejan de formar parte en su vertiente museística de nuestro objetivo. Comentario aparte merecen los estudios de Álvarez Urquieta, poseedor de una notable colección artística que años después venderá al propio museo, pues a través de sus conocimientos traza el primer discurso sobre la pintura colonial chilena y algunos de los pintores extranjeros precursores venidos tanto antes como inmediatamente después de la independencia, casos de Gil de Castro o Rugendas. De hecho, Romera $(1951,199)$ reconoce que "Su extenso trabajo, La pintura en Chile durante el periodo colonial constituye una introducción histórica a nuestra obra".

Así pues, los autores que decididamente impulsarán a nivel teórico la historiografía del arte en este país serán Álvarez Urquieta, Alfredo Benavides, Eugenio Pereira Salas y Antonio R. Romera, además de Cousiño, Mackenna, Lago y Vidor. De los que solo la mitad serán profesores universitarios o participarán activamente de la vida académica santiaguina. 


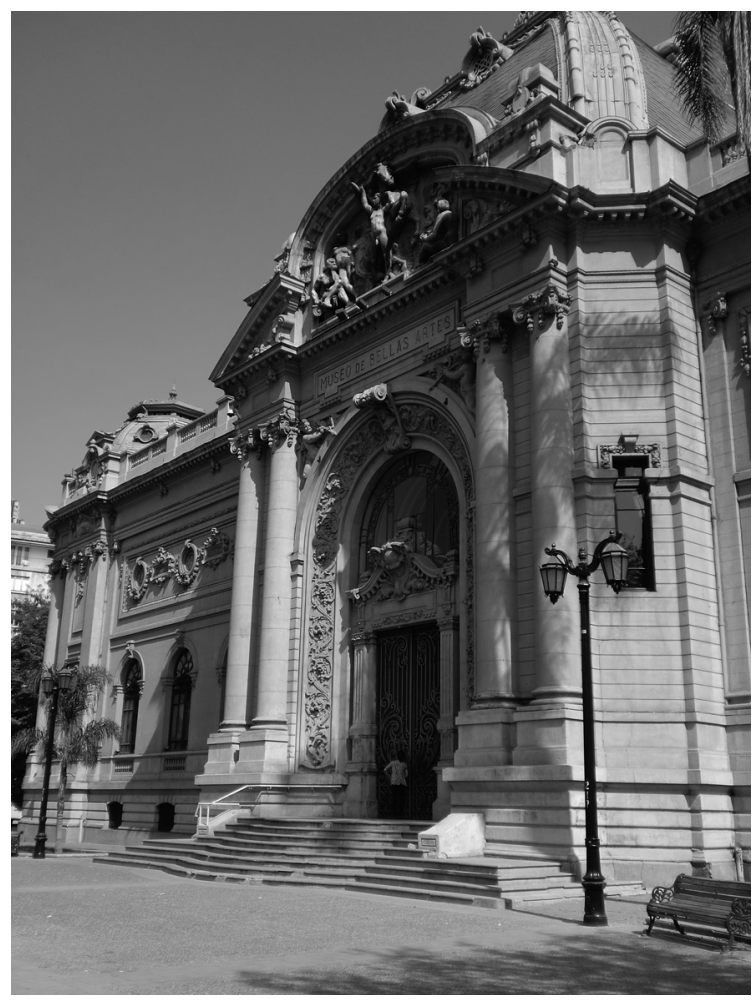

Fig. 1. Museo Nacional de Bellas Artes. Santiago (Fotografía $\mathrm{AF})$.

\section{HACIA UNA RENOVADA HISTORIOGRAFÍA DEL ARTE}

Consolidados tanto la Academia de Bellas Artes (1849) -Escuela a partir de 1858- como el Museo Nacional de Bellas Artes en su nuevo y definitivo emplazamiento diseñado por Jéquier (1910) (figuras 1 y 2), la teoría del arte chileno va creciendo al amparo de revistas especializadas o misceláneas de indudable interés -la Revista de Santiago, Las Bellas Artes, Revista Chilena, El Taller Ilustrado, Revista de Bellas Artes, Revista de Artes y Letras, ZigZag, Selecta, Los Diez o Bellas Artes, publicadas entre 1848 y 1928, serán algunas de ellas(Zamorano, 2013, 195-208, y Zamorano, Herrera, Madrid, Cortés, 2014, 291-309) y el impulso de las primeras generaciones de artistas que, formados en el extranjero, cimentarán una nueva plástica nacional con personalidad propia y definida. Circunstancias que generarán inevitablemente un nuevo clima cultural a través de los Salones, bien diferente del suscitado en la primera mitad del siglo XIX, pues los pintores foráneos no vendrán ya únicamente atraídos por la singularidad de su geografía, gentes y costumbres sino llamados por las más altas instancias oficiales para ocupar puestos de responsabilidad en las enseñanzas artísticas 


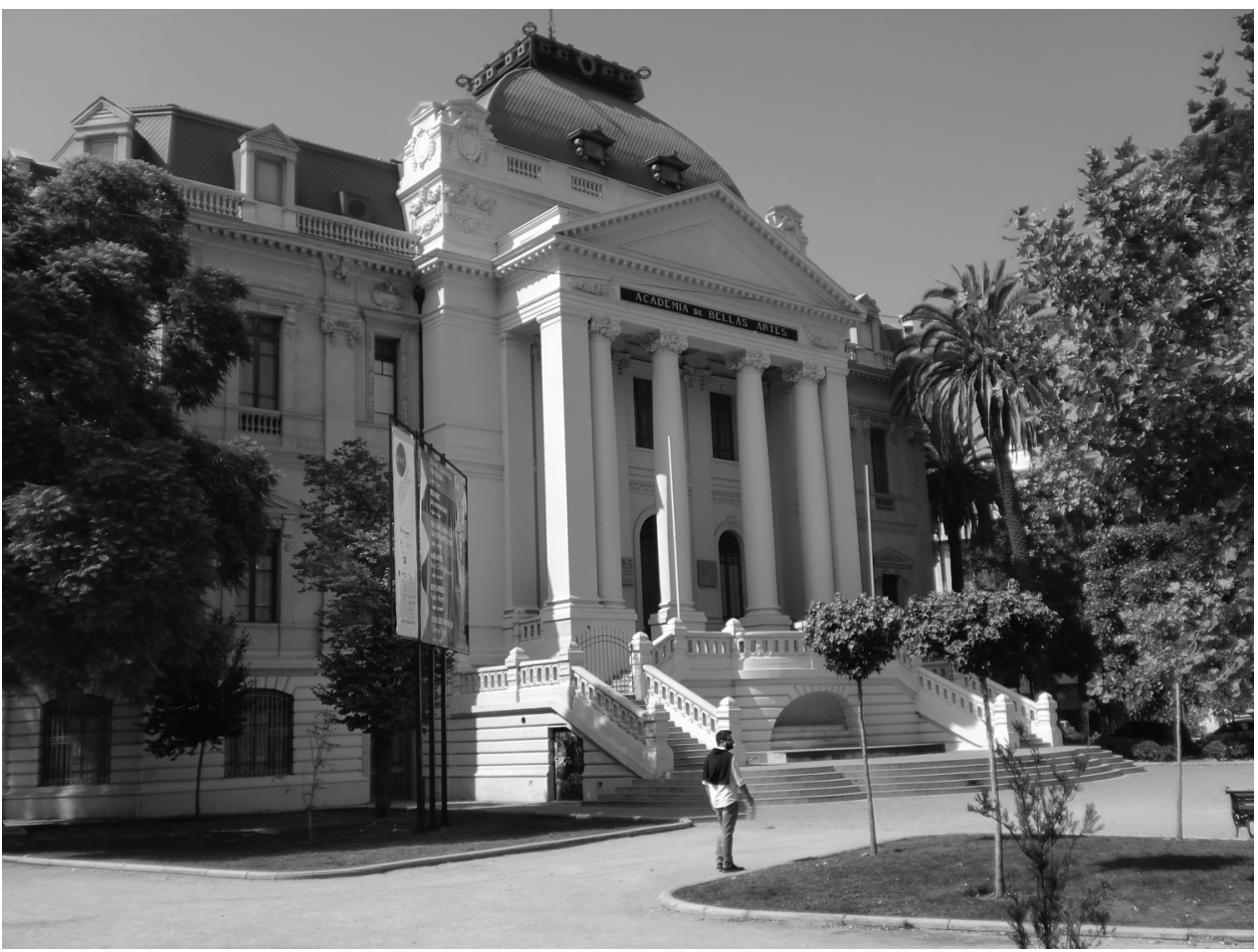

Fig. 2. Academia de Bellas Artes. Santiago (Fotografía AF).

y, como inevitable consecuencia, la potenciación y desarrollo de un determinado gusto. En esas precisas coordenadas cabe encuadrar la llegada de Cicarelli, Kirchbach, Mochi, Álvarez de Sotomayor o Vidor, entre otros.

En dicho período, más aún entre el último cuarto del siglo XIX y los primeros lustros del XX, tal como sucede en el resto del mundo occidental, Chile no será la excepción, los ojos de los artistas y de sus promotores van a estar centrados en París como centro neurálgico indispensable para la formación y transmisión de las ideas vanguardistas. Una y otra vez, los pintores y escultores chilenos son becados para llevar a cabo una estancia más o menos prolongada en la capital europea, convirtiéndose en avanzadillas privilegiadas que, junto a determinada élite económica, política y social muy activa del país, van a propiciar un progresivo acercamiento a sus gustos, cuando no la adopción directa de sus modelos, en detrimento del sustrato colonial.

Este proceso decididamente cosmopolita se evidencia con claridad, por ejemplo, en artistas como Blanco o Lira y en próceres como Rafael Errázuriz Urmeneta y Alberto Mackenna Subercaseaux, nacidos todos ellos entre 1839 y 1874. Especialmente en Lira, quien publicó en Las Bellas Artes de Santiago la traducción de la obra de Taine (1869) -solo cuatro años después de su edición en Francia-y, posteriormente, su Diccionario biográfico de pintores (1902); dos aportaciones que a la postre se convertirán en fundamentales para la 
historia del arte y su maduración posterior (De Nordenflycht, 2003, 34-38), aunque bien es cierto que el agudo criterio de Romera $(1951,200-201)$ recalca al referir con tiento y contundencia a la vez que:

La referencia a artistas chilenos es más bien escasa. Hubiera sido preferible que Lira dedicara su obra exclusivamente a las artes figurativas chilenas; su obra sería más útil (...) El libro de don Pedro Lira es por el contrario importantísimo para entrar en las ideas estéticas del maestro, para saber lo que pensaba del arte, para saber sus preferencias y aciertos críticos.

Contexto en el que las futuras aportaciones de Álvarez Urquieta, Benavides, Romera y Pereira Salas serán pioneras de un nuevo modo de hacer, de otra manera de entender el hecho artístico y sus complejas circunstancias (Ferrer, Madrid, 2015, 213-222).

\section{Luis Álvarez Urquieta (1877-1945)}

Personalidad poco conocida a pesar de su relevancia cultural en la primera mitad del s. XX y sensibilidad para con el arte, particularmente pictórico, de hecho poseía una gran colección artística que, finalmente, vendió al Museo Nacional de Bellas Artes. A pesar de no pertenecer al mundo académico e investigador, hizo gala de un conocimiento y sensibilidad nada frecuentes en el país, reflejados muy particularmente en tres de sus obras: La pintura chilena. Colección Álvarez Urquieta, Santiago de Chile, Imprenta la Ilustración, 1928; La pintura en Chile durante el período colonial, Santiago de Chile, Dirección General de Prisiones Imp., 1933; y El artista pintor José Gil de Castro, Santiago de Chile, Empresa periodística El Imparcial, 1934. Presentado el autor en su primer libro como "aficionado y coleccionista" por Onofre Jarpa, consta como miembro de la Academia Chilena de la Historia, institución que a su vez amparó la edición de sus textos en la década de 1930.

Sin manejar repertorios bibliográficos, por lo que adolecen todas ellas de falta de aparato crítico, no cabe duda que tienen el interés de ser pioneras en su género y, por consiguiente, el primer intento por tratar la evolución de la pintura en Chile, aunque solapadamente le sirvan a Álvarez Urquieta como excusa para basarse en su importante colección, toda vez que inmejorable catapulta para darla a conocer y revalorizarla en el contexto cultural de su tiempo (De Nordenflycht, 2003, 38-39), como finalmente sucedió a diferencia del altruismo de algunos de sus conciudadanos para con el citado museo. En palabras de Romera (1951, 199):

(...) Luis Álvarez Urquieta es el historiador más acucioso [y refiriéndose a su primera obra] (...) trabajo al que hay que acudir por la acumulación de datos y por el material gráfico (...) [aunque] (...) La interpretación de los problemas críticos y formales es casi nula, pero el mérito y el valor práctico de tales investigaciones son innegables.

\section{Alfredo Benavides Rodríguez (1894-1959)}

Arquitecto y profesor de la Universidad de Chile, hizo hincapié en la arquitectura de época colonial, además de otras reflexiones de carácter más general sobre esta materia. Según Solanich $(2013,25)$ : 
[Junto a Pereira] Investigadores de fuste, regocijados por las manufacturas de los siglos coloniales XVI, XVII y XVIII, no trepidan en hurgar archivos y bibliotecas; viajar visitando sitios, pueblos y ciudades que ostentan el patrimonio que les apasiona, ocupa y anhelan salvaguardar (...) Los dos investigadores chilenos dotan a la historia del arte de rango superior y demuestran en sus trajines una vocación de laboriosidad minuciosa y prolija (...).

Ejemplo de ello son La arquitectura a través de la Historia, Santiago de Chile, Establecimientos gráficos "Balcell \& Co.", 1930; Historia de la arquitectura, 2 tomos, Santiago de Chile, 1941; o La arquitectura en el Virreinato del Perú y en la Capitanía General de Chile, Santiago de Chile, Ercilla, 1941, reeditada en dos ocasiones, la cual es reseñada por Solanich (2013, 28-29). En palabras de Guarda (1965, 5-6), recogidas en su discurso de incorporación a la Academia Chilena de la Historia el 5 de junio de 1965:

La alta calidad científica de sus obras mereció a su autor reconocimiento de parte de distinguidas instituciones (...) [pues] contaba don Alfredo Benavides con una formación esmerada, cultivada en numerosos viajes (...) visitados siempre con un serio criterio de estudioso.

\section{Antonio Rodríguez Romera (1908-1975)}

Crítico, historiador y teórico del arte toda vez que caricaturista español, llegó a Chile en 1939 como exiliado a consecuencia de la guerra civil, aunque en aquel momento se encontraba en París. Establecido y domiciliado en Santiago desarrolló una intensísima actividad cultural, en especial vinculada a la pintura chilena contemporánea (Zamorano, Cortés, Muñoz, 2003, 41-53), siendo reconocido como “(...) de los granados pilares de la pintura chilena, con porte estricto y excelencia sin par" (Solanich, 2013, 31). Entre sus numerosos libros se pueden encontrar obras dedicadas a Da Vinci, Rubens, Rembrandt, Van Gogh, Matisse, Mori, Valenzuela Puelma, destacando sobremanera Historia de la pintura chilena, Santiago de Chile, Editorial del Pacífico, 1951, reeditada en diversas ocasiones y primer intento verdaderamente moderno para su análisis, estructuración y difusión. Ambicioso estudio redactado con una gran soltura literaria y conocimiento de causa, dividido en una introducción (9-15), nueve capítulos que abarcan desde los precursores y sus seguidores (1752) hasta el Grupo de Montparnasse, seguidores y movimientos afines (183-196), la bibliografía consultada y comentada (197-212) y un índice onomástico (213-219), además del índice correspondiente (221-223) y numerosas ilustraciones en blanco y negro. Las conclusiones a las que llega Romera $(1951,11)$ ya nos las va anticipando desde las primeras líneas al afirmar taxativamente:

Existe una pintura chilena (...) Lo que queda por determinar es su naturaleza [añadiendo a continuación que] (...) las artes figurativas nacionales son plurales y diversificadas [pues] (...) los artistas de Chile no podían permanecer ajenos a los influjos que llegaban de fuera.

Para proseguir lúcidamente con reflexiones del siguiente tenor:

La pintura más cercana, cualquiera que sea su característica esencial, no abandona el tema del paisaje. Al contrario, en muchos casos es pretexto para realizar una obra en la cual el color de la naturaleza chilena se nimba de lírica belleza. Tenemos que el paisaje es, pues, 
una constante. Pero este rasgo atañe al tema, no a la pintura en sí [aseverando que la pintura chilena se aproxima al] (...) predominio del color, de las formas esfumadas, de la expresividad (Romera, 1951, 13-14).

Razonamientos que, como en cascada, van definiendo magistralmente párrafo a párrafo lo que nunca se había dicho hasta entonces por ningún teórico del arte: "La pintura nacional sigue una curva interior, oculta, de exaltado individualismo (...) Pero en lo más profundo cada artista conserva una indeclinable, una insobornable independencia espiritual" (Romera, 1951, 14), hasta que compone novedosamente la osamenta de la pintura en Chile en:

(...) precursores y sus inmediatos secuaces (...) el romanticismo paisajista (...) la pléyade de la Academia de Pintura (...) Pedro Lira, Alfredo Valenzuela Puelma, Alberto Valenzuela Llanos y Juan Francisco González [“los cuatro maestros”] (...) la pléyade de 1913 [a la sombra de Álvarez de Sotomayor] (...) la prolongación de las normas del objetivismo finisecular (...) los independientes, por su autonomía estética, y el grupo de Montparnasse (...) (Romera, 1951, 14-15).

Además de la labor de Antonio R. Romera como crítico artístico, “(...) con él se inicia verdaderamente la crítica de arte en Chile" (Zamorano, Cortés, Muñoz, 2005, 136), no cabe duda de que:

(...) estableció un modelo de análisis para la pintura nacional. Éste consideró una doble mirada. De una parte, determinó claves y constantes, es decir, propuso una forma de organización conceptual, que intenta definir el carácter de nuestra plástica a partir de sus orientaciones y caracteres específicos; de otra, plantea una estructuración de desarrollo cronológico, cuyo mérito fue haber ordenado, orientado y clasificado las distintas tendencias en la pintura nacional republicana. Y todo ello en un medio de escasa tradición teórica y crítica, donde prácticamente todo estaba por hacer. Por ello, estimamos su obra como pionera en el contexto de la pintura nacional (...) [concluyendo que Romera] Es un ecléctico (Zamorano, Cortés, Muñoz, 2005, 136-137).

Cabe decir que los datos aportados por Romera, concretamente los referidos a la Generación del 13, son ampliados por el pintor Waldo Vila (1894/97-1979) en Una capitanía de pintores, Santiago de Chile, Editorial del Pacífico, 1966, dedicado de alguna manera en el colofón a Pablo Neruda -autor del prólogo-; obra en la que hace gala de haber participado en la misma, a pesar de que confiesa “(...) no pertenezco a escuela alguna, ni corrillo o cenáculo determinado" (Vila, 1966, 10), aunque a decir verdad no guarda (ni siquiera lo pretende) relación con el trabajo científico de Romera, Pereira, etc. Se trata más bien de una proyección de su faceta de crítico artístico, como una recopilación de trabajos periodísticos, pues conserva esa frescura e inmediatez; tal cual sucede con Pintura joven. La década emergente, publicada en 1973 por la misma editorial y ciudad.

\section{Eugenio Pereira Salas (1904-1979)}

Historiador de la Cultura es como mejor se podría definir la labor que ejerció durante su vida Pereira Salas, no en vano, desde su formación humanística cimentada en Chile, Europa y los EEUU, Pereira Salas abordó no pocos de los aspectos característicos de la 
cultura chilena a partir de tres coordenadas: la vida cotidiana, las artes escénicas y el arte en el siglo XIX. Así por ejemplo, se introdujo en la cocina, los juegos o el folklore; el teatro y la música; o la arquitectura, la escultura y la pintura producidas en su país natal (De Nordenflycht, 2003, 37-38).

Tal vez por ello, el más completo por diverso de todos los precursores de cuántos nos hemos ocupado hasta el momento, en quien confluyeron los nuevos derroteros por los que transitaban las diversas corrientes historiográficas gracias a sus estancias en el exterior. Fruto de ello, en cuestiones propiamente artísticas, fue su obra Historia del Arte en el Reino de Chile, Santiago, Universidad de Chile, 1965; un gran estudio en el período en el que vio la luz y que, de alguna manera, recogía el testigo de Álvarez Urquieta en materia colonial, toda vez que ampliaba sustancialmente y dotaba de aparato crítico el acervo aportado por aquel en el primer tercio del siglo XX. De hecho, el libro se divide en dieciocho capítulos que abarcan un lapso cronológico tan amplio como el comprendido entre 1541 y 1812 (327 páginas), es decir casi tres siglos, aderezado de notas (329-358), numerosas láminas en color y blanco y negro (359-486) e índices alfabéticos y geográficos (487-497). En palabras de Solanich $(2013,31)$ :

Huelgan comentos a la escrupulosa pesquisa y los precedentes que proporcionan su magno tesón, que aviva el afecto por el arte colonial. El cúmulo de hechos, datos, cifras, nombres e inventarios, ceñidos a estrictas fuentes documentales, enlazan un mamotreto capital, todavía sin parangón.

Académico de la Universidad de Chile, institución en la que coincidió con Lago y Benavides, tuvo una dilatada actividad docente e investigadora, llegó a presidir entidades e instituciones culturales del país como la Academia Chilena de la Historia, obteniendo asimismo en 1974 el Premio Nacional de Historia. Póstumamente (1992) fue editado un libro recopilatorio con el título Estudios sobre la Historia del Arte en Chile Republicano.

\section{EL MUSEO NACIONAL DE BELLAS ARTES COMO DINAMO HISTORIOGRÁFICO}

Otro hito para el crecimiento y la consolidación de la historia del arte en Chile, complementario e interdependiente de otros procesos relativos a la gestión cultural y la labor editorial será, como ya referenciamos en su momento, el Museo Nacional de Bellas Artes (antaño Museo Nacional de Pintura), que verá paulatinamente acrecentada su colección inicial mientras estuvo radicado en el Congreso (1880) y el Partenón de Quinta Normal (1887) hasta su definitiva ubicación en el nuevo edificio del Parque Forestal (1910) (figura 3), mediante donaciones o adquisiciones y que necesitará de su catalogación progresiva. Por tanto, se convierte en generatriz de nuevos estudios que, nacidos a su sombra, confluirán con su propósito a la par que con el desarrollo de la historiografía del arte nacional (Ferrer, Madrid, 2016, 141-151). 


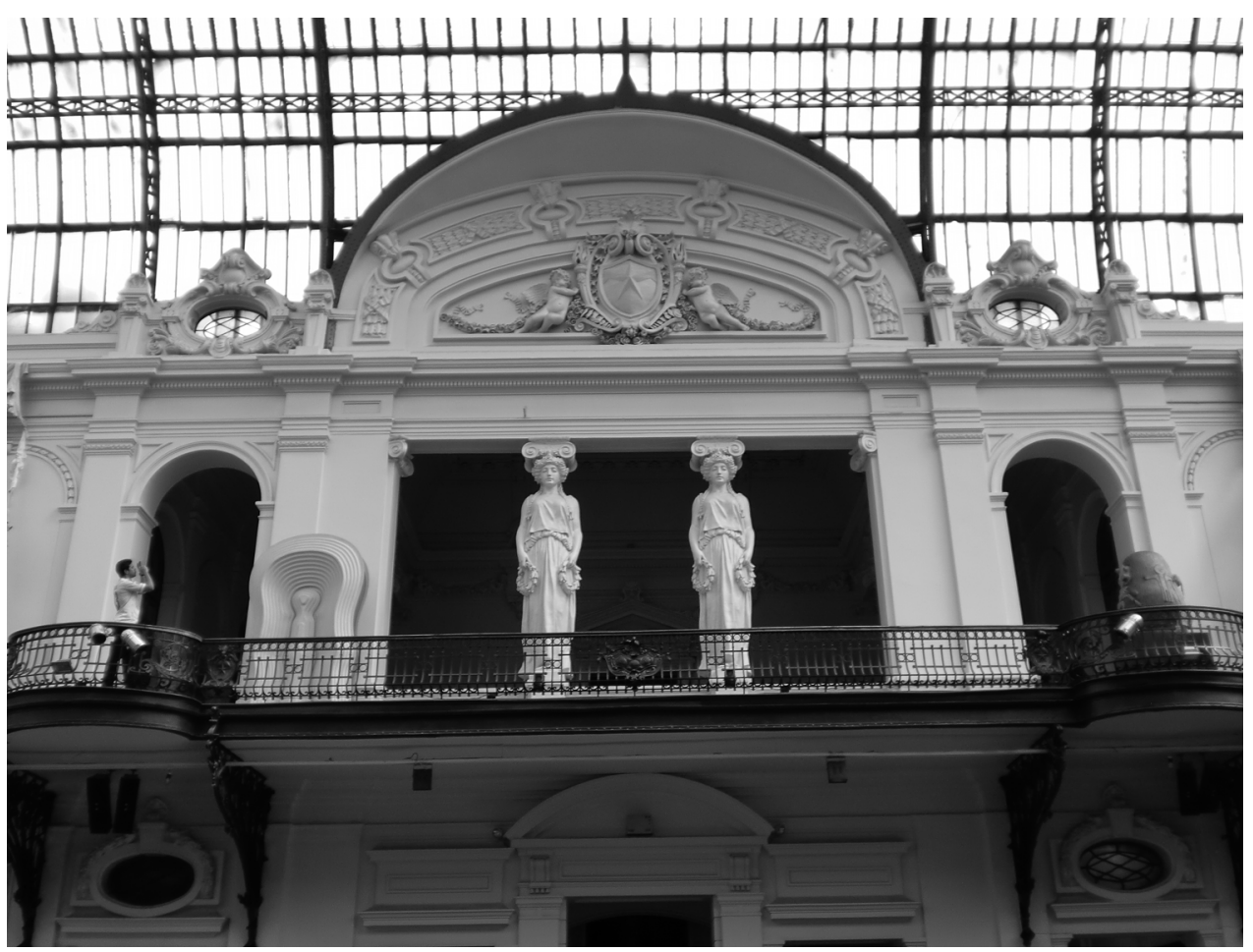

Fig. 3. Museo Nacional de Bellas Artes, interior. Santiago (Fotografía AF).

\section{Luis Cousiño Talavera (1874-1948)}

Quinto director del Museo Nacional de Bellas Artes, desempeñó su cometido entre 1923 y 1926, aunque el único testimonio público que ha quedado de su paso es el Museo de Bellas Artes. Catálogo general de las obras de pintura, escultura, etc., Santiago de Chile, Soc. Imprenta y Litografía Universo, 1922, donde firma como miembro del Consejo de Bellas Artes. Visto con perspectiva, el primer intento por catalogar las piezas que se fueron depositando en el museo, además de proporcionar una breve biografía de sus artífices siguiendo, quizás, el Diccionario de Lira. Un trabajo fundamental para determinar cuáles eran las obras, sus autores, el tipo de adquisición en cada caso o su depósito fuera del mismo hasta ese momento y sobre el que incidió la obra de T. Lago y P. Vidor ocho años después. Siendo al poco tiempo de su edición aumentada con otras adquisiciones, en especial la Colección de Álvarez Urquieta.

La obra parte con la información consultada al alcance del autor, las actas del museo de 1904 y anteriores, transmisoras de escasas o nulas noticias según su testimonio (3-5); sigue con la distribución de los autores por salas acompañadas de la numeración de los cuadros (7-16) y los planos del recinto museístico, para pasar a la parte más sustancial del mismo: la Galería Lillo a través de 124 pinturas, dibujos, aguafuertes, etc., y 142 libros sobre materias 
artísticas (17-158); los cuadros que se hallan guardados o en depósito (159-173); los que se encontraban fuera del museo (174-178); la relación de sus esculturas, seguramente las que trajo de Europa Alberto Mackenna y pequeños objetos en vitrinas no clasificados; otras esculturas y objetos artísticos (180-222) y, por último, más esculturas y relieves guardados en la Escuela de Bellas Artes (223). Un intento de catalogación que necesitará ser revisada pocos años después, como ya se ha referido, y que se convierte en un hito sobre el que -suponemos- pivotarán las obras que inicialmente comenzaron a formar parte de las colecciones del museo capitalino desde 1880 hasta 1922.

\section{Alberto Mackenna Subercaseaux (1874-1952)}

Sobrino de Benjamín Vicuña Mackenna, fue como su tío intendente de Santiago entre 1927-1929 además de hombre de negocios. En 1901 se le comisionó por el gobierno para adquirir en Europa un "museo de copias", es decir reproducciones de las obras cumbres de la estatuaria grecolatina y muebles. Al regresar con la colección y esta no disponer de un lugar donde ser exhibidas fueron almacenadas. Fue impulsor del Museo y de la Escuela de Bellas Artes, del que se convirtió en su décimo director entre 1933-1939, y previamente comisarió la exposición universal con que se inauguró el recinto en 1910.

Con ocasión de su periplo europeo publicó Luchas por el Arte (1915), en el que recoge una conferencia pronunciada en el Ateneo de Santiago, "El origen del 'Museo de copias"” (22 de mayo de 1899), una carta abierta al ministro de Instrucción pública, "Un futuro Museo de Bellas Artes" (9 de enero de 1901), y un artículo de opinión, "Utilidad de un museo del arte industrial" (1901), además de otros textos sobre el particular y autores. No cabe duda que, como refiere Madrid (2012, 61 y 63), a través de sus tres escritos "El primer viaje se podría connotar como el de la experiencia y el deseo [de producción de infraestructuras], luego el segundo es el de la materialización [proyección] (...) [donde] resume un sentir de época, el de vincular el desarrollo de la formación artística al ámbito industrial y del gusto al progreso". En suma, un libro que recoge, entre otras muchas anécdotas de indudable interés para la historia del arte en Chile, el sentir de un hombre ilustrado en las coordenadas espaciotemporales que hubo de transitar.

\section{Tomás Lago Pinto (1903-1975)}

Escasamente considerado por la historiografía artística chilena, Tomás Lago se erige en una personalidad que merece una mayor atención por formar parte activa de un período trascendental para el arte y la literatura de Chile. Muy cercano a Pablo Neruda, este ñublense nacido en Chillán ejerció un papel bien destacado en la reivindicación de la cultura tradicional y la artesanía del país a través de libros como Exposición americana de artes populares, Santiago de Chile, Universidad de Chile, 1943, y Arte popular chileno, Santiago de Chile, Col. Imagen de Chile, Ed. Universitaria, 1971, como también en las llamadas artes mayores, especialmente en lo que concierne a la pintura, gracias a obras como $E l$ Museo de Bellas Artes: 1880-1930, Santiago de Chile, Universidad de Chile, 1930, que viene a complementar y hasta aclarar -con sus textos y los de Pablo Vidor- la de Cousiño editada unos años atrás, pues dividida en tres grandes bloques: "Precursores de la pintura 
chilena", de Lago (5-35), las ilustraciones escogidas (37-90) y "El Museo de Bellas Artes", de Vidor (91-107) que conjugan discurso, imagen e historia del museo y una nueva catalogación en la que aparecen como benefactores, además de Lillo, Carlos Cousiño, Francisco Echáurren Huidobro, Agustín Edwards, María Luisa Lynch de Gormaz, María Luisa Mac-Clure de Edwards, Carlos García Huidobro, Ramón Subercaseaux, Joaquín Fabres, monseñor Ignacio Víctor Eyzaguirre, Charles Francis Browne, el general Marcos Maturana, Alfredo Concha Vicuña, Pedro Lira, Vicente M. Carrió, la familia de Federico W. Schwager, Francisco Pons Arnau, Angelo Sonmarruga, la Bolsa de Comercio de Santiago, Enrique Lynch, Alberto Orrego Carvallo, Alejandro Eguigúren, la Universidad de Chile, Federico Casas Basterrica, Laura M. de Saridakis, Pedro Felipe Íñiguez, Benjamín F. Bernstein, Candelaria Bazo Gallo, Juan de Dios Vargas, Adela Ugarte de Bianchi Tupper, Arturo Blanco, Richard Richon-Brunet, José Perotti y Guillermo Vergara; Cerámica de Quinchamalí, Santiago, Universidad de Chile, 1958. Obras todas ellas que se completan con las dedicadas a Julio Ortiz de Zárate, Santiago de Chile, Col. artistas chilenos, Universidad de Chile, 1956; o a Rugendas. Pintor romántico de Chile, Santiago, Universidad de Chile, 1960, reeditada con posterioridad.

Docente en la Facultad de Bellas Artes de la Universidad de Chile, es uno de los pocos profesores e investigadores que imprimen un método científico a sus trabajos. No en balde sus textos están dotados de una mayor clarividencia narrativa, conceptual y decididamente heurística. Impulsó el Museo de Arte Popular Americano (MAPA) en Santiago, a la postre el primero de su carácter en el continente. Espacio cultural del que también fue director hasta 1968.

\section{Pablo Vidor Doctor (1892-1991)}

Pintor de origen húngaro que, después de formarse en Budapest, Viena, Múnich y Berlín se trasladó a Chile, donde se nacionalizó en 1931. Desde 1928 fue profesor de la Escuela de Bellas Artes, convirtiéndose después en el noveno director del Museo Nacional de Bellas Artes, cargo que ejerció entre 1930 y 1933. También ejerció la crítica de arte en diversas publicaciones, siendo la relativa al museo la que más nos interesa y que hemos abordado al tratar de Tomás Lago.

\section{CONCLUSIÓN}

En resumen, algunos de los teóricos del arte estudiados, sobre todo los que tienen una vertiente universitaria más definida, van a sentar cátedra y sus líneas de investigación serán las que -con las primeras promociones de historiadores del arte a mediados de los años '70 del pasado siglo- se irán desarrollando en Chile sin solución de continuidad. Sin embargo, su fructífera labor historiográfica, en líneas generales, sigue siendo un referente incuestionable en nuestros días y sus métodos, aportaciones y resultados un campo abonado sobre los que se cimentará la disciplina. De hecho, sus obras en el mayor de los casos todavía suscitan el interés de quienes las consultan, atendiendo que partieron de una realidad bibliográfica tan difusa como inconstante, relativamente tardía y dispersa en multitud de trabajos en publicaciones culturales de vida más o menos efímera. 
Por tanto, el hecho de que entre 1928, año en el que salió a la luz la obra de Álvarez Urquieta, La pintura chilena. Colección Álvarez Urquieta, y 1965, en el que se publicó la de Pereira Salas, Historia del Arte en el Reino de Chile, surgieran los primeros estudios dedicados exclusivamente al arte chileno, con criterios afines a la historiografía del arte contemporánea, por un elenco de autores como los anteriormente citados, confirma que en dicho lapso cronológico se cimentaron las grandes líneas maestras del arte chileno: colonial, republicano y contemporáneo. Directrices que, a otro ritmo y exigencia, siguieron de alguna manera quienes se ocuparon de las colecciones del Museo Nacional de Bellas Artes de Santiago, espacio cultural de primera magnitud que, todavía hoy, está en deuda con las aportaciones de Cousiño, Mackenna, Lago y Vidor dada la escasa actividad editorial, investigadora y difusora de sus fondos artísticos hasta nuestros días.

\section{BIBLIOGRAFÍA}

ÁLVAREZ, Luis (1928): La pintura chilena. Colección Álvarez Urquieta, Santiago de Chile, Imprenta la Ilustración, 54 p.

ÁLVAREZ, Luis (1933): La pintura en Chile durante el período colonial, Santiago de Chile, Dirección General de Prisiones Imp., 80 p.

ÁlVAREZ, Luis (1934): El artista pintor José Gil de Castro, Santiago de Chile, Empresa periodística El Imparcial, $50 \mathrm{p}$.

BENAVIDES, Alfredo (1930): La arquitectura a través de la Historia, Santiago de Chile, Establecimientos gráficos "Balcell \& Co.", 219 p.

BENAVIDES, Alfredo (1941-1943): Historia de la arquitectura. 2 tomos, Santiago, Galcon, $180+$ $249 \mathrm{p}$.

BENAVIDES, Alfredo (1941): La arquitectura en el Virreinato del Perú y en la Capitanía General de Chile, Santiago de Chile, Ercilla, 358 p.

COUSIÑO, Luis (1922): Museo de Bellas Artes. Catálogo general de las obras de pintura, escultu$r a$, etc., Santiago de Chile, Soc. Imprenta y Litografía Universo, 222 p.

FERRER, Albert, MADRID, Alberto (2015): "Los precursores de la historia del arte en Chile", Ars Longa, $\mathrm{n}^{\circ} 24,213-222$.

FERRER, Albert, MADRID, Alberto (2016): "The National Museum of Fine Arts of Santiago and its publications: 1922-2009", Universum, año 31, vol. 1, 141-151.

GUARDA, Gabriel OSB (1965): "Santo Tomás de Aquino y las fuentes del urbanismo indiano", Boletín de la Academia Chilena de la Historia, $\mathrm{n}^{\circ}$ 72, 5-50.

LAGO, Tomás (1943): Exposición americana de artes populares, Santiago de Chile, Universidad de Chile, $221 \mathrm{p}$.

LAGO, Tomás (1956): Julio Ortiz de Zárate, Col. artistas chilenos, Santiago de Chile, Universidad de Chile, 18 p.

LAGO, Tomás (1958): Cerámica de Quinchamalí, Santiago, Universidad de Chile, 57 p.

LAGO, Tomás (1960): Rugendas. Pintor romántico de Chile, Santiago, Universidad de Chile, 198 p.

LAGO, Tomás (1971): Arte popular chileno, Santiago de Chile, Col. Imagen de Chile, Ed. Universitaria, $136 \mathrm{p}$.

LIRA, Pedro (1902): Diccionario biográfico de pintores, Santiago de Chile, Litografía Esmeralda, $551 \mathrm{p}$.

MACKENNA, Alberto (1915): Luchas por el Arte, Santiago-Valparaíso, Soc. Imprenta-Litografía "Barcelona", 103 p.

MADRID, Alberto (2012): “Alberto Mackenna Subercaseaux: Viajero ilustrado", en Guzmán, Fernando, Martínez, Juan Manuel (ed.), Vínculos artísticos entre Italia y América. Silencio historiográfico. VI Jornadas de Historia del Arte, Santiago de Chile, 55-66. 
NORDENFLYCHT CONCHA, José de (2003): "Territorio, filiación y texto: de la historia del arte en Chile a la historia del arte chileno", en Guzmán, Fernando, Cortés, Gloria, Martínez, Juan Manuel (comp.), Iconografía, identidad nacional y cambio de siglo (XIX-XX). Jornadas de Historia del Arte en Chile, Santiago, RIL Editores, 31-40.

PEREIRA, Eugenio (1965): Historia del Arte en el Reino de Chile, Santiago, Universidad de Chile, $497 \mathrm{p}$.

PEREIRA, Eugenio (1992): Estudios sobre la Historia del Arte en Chile Republicano, Santiago, Ed. Regina Claro Tocornal, Ediciones de la Universidad de Chile, 344 p.

ROMERA, Antonio (1951): Historia de la pintura chilena, Santiago de Chile, Editorial del Pacífico, $222 \mathrm{p}$.

SOLANICH, Enrique (2013): Escritos de Arte en Chile. Breviario de textos, Santiago de Chile, Aica, 249 p.

VILA, Waldo (1966): Una capitanía de pintores, Santiago de Chile, Editorial del Pacífico, 197 p.

VILA, Waldo (1973): Pintura joven. La década emergente, Santiago de Chile, Editorial del Pacífico, $109 \mathrm{p}$.

ZAMORANO, Pedro Emilio (2005): "El español Antonio Romera y la historiografía artística chilena: una propuesta de organización fundacional", Archivo Español de Arte, vol. 78, n 310, 133-144.

ZAMORANO, Pedro Emilio (2013): "El Taller Ilustrado: periódico de artistas y para artistas", Aisthesis, n $54,195-208$.

ZAMORANO, Pedro Emilio, CORTÉS, Claudio, MUÑOZ, Patricio (2003): "Semiótica e historia del arte: el caso de Antonio Romera en Chile (1940-1975)”, en Guzmán, Fernando, Cortés, Gloria, Martínez, Juan Manuel (comp.), Iconografía, identidad nacional y cambio de siglo (XIXXX). Jornadas de Historia del Arte en Chile, Santiago, RIL Editores, 41-53.

ZAMORANO, Pedro Emilio, HERRERA, Patricia, MADRID, Alberto, CORTÉS, Claudio (2014): "Circulación de la información y la reflexión artística en Chile: Panorama de las revistas desde 1900 hasta la década del sesenta", Universum, vol. 29, n² 2, 291-309. 
